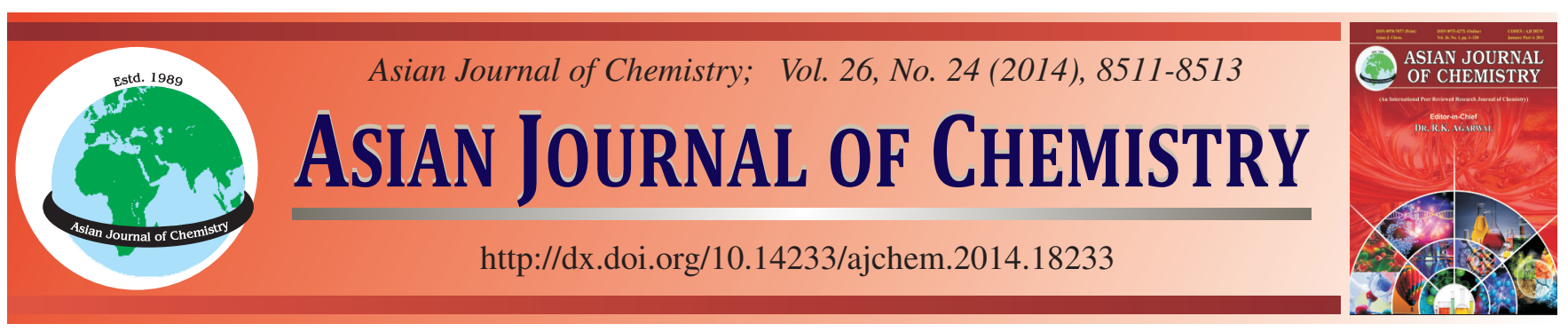

\title{
Respond Characteristic Property in Different Temperatures of SAW Sensor with $p$-Tertbutylcalix[4] Coatings to Detect Sarin
}

\author{
BingQing CAO*, Qibin HuAng and Molin Qing
}

State Key Laboratory of NBC Protection for Civilian, Beijing 102205, P.R. China

*Corresponding author: Fax: +86 10 69760246; Tel: +86 10 66758621; E-mail: caobingqing961@ @ohu.com

Received: 2 June 2014;

Accepted: 12 August 2014;

Published online: 1 December 2014;

AJC-16392

\begin{abstract}
25-(Thioalkyl-alkoxy)-p-tertbutylcalix[4]arene self-assembly molecular imprinted film as the sensitive film was coated on the gold delay line of SAW sensors to detect the orgnaophosphorous analogue compounds such as sarin. The sensor has special response to organoosphorus compounds and the response frequency shift of this sensor to organosphorus compounds. The possible explanation of interactions between the coatings and organosphorus compounds is discussed. The results indicated that the SAW sensor coated with self-assembled monolayers character had high sensitivity to sarin, faster response, repetition and had important reference value in future practical application.
\end{abstract}

Keywords: Calixarene, Surface acoustic SAW sensor, Self-assembly, Sarin, Detection.

\section{INTRODUCTION}

At present, chemical terrorism and antiterrorism struggle are gradually austere, rapidly accurate detecting harmful substances in the environment becomes an important problem for all the countries in the world. Surface acoustic wave sensor has been prone various merits such as integrated rization, intellectualized, being able to realize remote detecting because whose volume is small, selectivity is high, cost is low, sensitiveness is high. It has become a kind new and developing sensor of detecting chemical poison gas and the explosive, having been paid close attention to broadly in recent years. The aspects such as synthesis, coating method and application study of sensitive film material have all got very big progressing at full speed development ${ }^{1-4}$.

The request of organophosphorus, organic sulphur toxic agent or related compouds to film material of SAW sensor is very harsh, film material reshaping studying, synthesis of sensitive film material and other aspects such as raising film material sensitiveness or stability, removing disturbance are in progress to the significance doing the research and going a step further, studies of military affairs on developing new model sensitive film material have important practical significance especially. Zhao et al..$^{5}$ made use of the SAW sensor based on self-assembly polydiacetylene calixarene derivatives bilayer film in gold surface to detect the chlorobenzene, toluene and others aromatic organics. The results indicated that the upper margin functional groups of calixarenes have important contributions to sensitiveness and selectivity. Ghidini et al. ${ }^{6}$ termi- nation modified thioether resorcin[4]arene derivatives, a selfassembly monomolecular membrane, are used to check four chloroethylene, whose sensitiveness can reach nanogram grade.

In this article, methyl hydroxy phosphonic acid isopropyl ester (1) (Fig. 1) was taken as the template molecule, 25-(thioalkylalkoxy)-p-tertbutylcalix[4]arene (2) (Fig. 1) self-assembly molecular imprinted film as the sensitive film was used in acoustic surface wave of chemical sensor and coated film on the gold delay line of SAW sensors to detect the orgnaophosphorous analogue compounds such as sarin. The response characteristic of the film to sarin was studied and a good result was acquired.

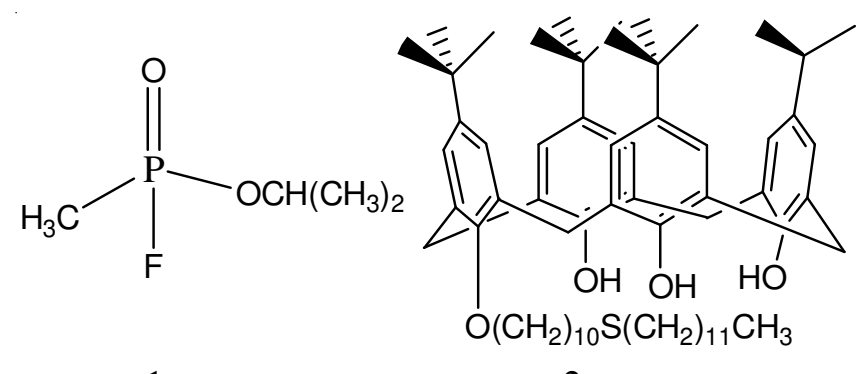

1

Fig. 1. Structure of methyl hydroxy phosphonic acid isopropyl ester and 25-(thioalkyl-alkoxy)-p-tertbutylcalix[4]arene

\section{EXPERIMENTAL}

25-(Thio-alkyl alkoxyl)-p-tert-butyl calixarene was synthetized in our laboratory. Sarin, methyl hydroxy phosphonic acid 
isopropyl ester were reagent grade (Research Institute of Chemical Defence, China). The other chemicals were reagent grade (Peking Chemical Reagent, Peking, China).

For the instrumentations, a surface acoustic wave double delay line (Acoustics Institute of Chinese Science, the center frequency $300 \mathrm{MHz}$, delay the line as the area $4 \mathrm{~mm}^{2}$ gold film), a Model Proteck C3100 Frequency Mter, (Korean Proteck company), a Model FHG02 toxic agent detect instrument (Peking, Peking analyzes instrument factory), a model FHD01 gas dynamic generator (Research Institute of Chemical Defence, China) were used in this article.

Preparation of self-assembly imprint film of SAW sensors: $0.0099 \mathrm{~g}$ (0.01 mmol) 25-(thioalkyl-alkoxy)- $p$ tertbutylcalix[4]arene was dissolved in $100 \mathrm{~mL}$ of mixed solution (acetone and ether, $\mathrm{v} / \mathrm{v}=1: 1$ ) into $0.0001 \mathrm{~mol} / \mathrm{L}$ selfassembly universe solution, added $100 \mathrm{~mL}$ of $10^{-5} \mathrm{~mol} / \mathrm{L}$ methyl hydroxy phosphonic acid isopropyl ester solution, standing for $12 \mathrm{~h}$ to be used behind.

The surface acoustic wave double delay line surface was purged with $\mathrm{v}$ (sulphuric acid): $\mathrm{v}$ (hydrogen peroxide) $=3: 1$ Piranha solutions, then the gold SAW delay line was washed with anhydrous alcohol and deionized water three times to bright and clean, with high purity $\mathrm{N}_{2}$ to puff surface foreign substance.

Then the above-mentioned surface acoustic wave double delay line had been soaked in the above-mentioned selfassembly universe solution for $24 \mathrm{~h}$, after taken out, was washed with acetone, anhydrous alcohol and deionized water, respectively five times to eliminate methyl hydroxy phosphonic acid isopropyl ester, the template molecule and treated as before steps. Its fundamental frequency and strepitus were measured at the end.

\section{RESULTS AND DISCUSSION}

Influences of temperature to calixarene-SAW sensor: When temperature changed, substrate material in SAW sensor would generate expansion caused by heat and contraction caused by cold and acoustic propagation path would change. Base coefficient of elasticity, density, dielectric constant and piezoelectric modulus changed at the same time. The wave velocity was affected by these changes, therefore the performance index and effectiveness of SAW sensor was often influenced by the temperature changes. At meantime, the temperature step up returned the space structure of the sensitive film surface partly and the feasible film with molecule interlinking descended, ultimately leading to that the respond frequency, sensitiveness, recovery time of a sensor would be diversified. Under the different temperature condition, calixarene-SAW sensors were used to detect the same concentration sarin, experimental results were listed in Table- 1 .

The results indicated that in the same concentration sarin, with the ambient temperature rising gradually, the respond time of SAW-MIP-sarin sensor became more and more shortly, corresponding lessening of signal, but rehabilitated also more and more quickly to sarin.

In isothermal Langmuir adsorption model, adsorb coefficient a changes following temperature and adsorption heat, whose relational expression is represented by the formula $\mathbf{1}$ :

\begin{tabular}{cccc}
\hline \multicolumn{5}{c}{ TABLE-1 } \\
DETECTION RESULT OF SAW SENSOR \\
TO SARIN IN DIFFERENT TEMPERATURE \\
\hline $\begin{array}{c}\text { Temperature } \\
\left({ }^{\circ} \mathrm{C}\right)\end{array}$ & $\begin{array}{c}\text { Respond frequency } \\
(\mathrm{KHz})\end{array}$ & $\begin{array}{c}\text { Respond time } \\
(\mathrm{min})\end{array}$ & $\begin{array}{c}\text { Resume } \\
\text { time }(\mathrm{s})\end{array}$ \\
\hline 15 & 2.65 & 3.4 & 100 \\
20 & 2.15 & 2.9 & 80 \\
25 & 1.78 & 2.5 & 65 \\
30 & 1.35 & 2.0 & 40 \\
40 & 1.03 & 1.5 & 30 \\
50 & 0.79 & 0.8 & 18 \\
\hline
\end{tabular}

$$
\mathrm{a}=\mathrm{a}_{\mathrm{O}} \mathrm{e}^{\frac{\mathrm{Q}}{\mathrm{RT}}}
$$

when after taking logarithms the formula 2 was put into the formula $\mathbf{1}$, the formula 2 was obtained

$$
\ln \left(\frac{\Delta \mathrm{F}}{\Delta \mathrm{F}_{\mathrm{m}}-\Delta \mathrm{F}}\right)=\ln \mathrm{a}_{0}+\ln \mathrm{C}+\frac{\mathrm{Q}}{\mathrm{RT}}
$$

In the experiment, $\mathrm{s}=\frac{\Delta \mathrm{F}}{\Delta \mathrm{F}_{\mathrm{m}}-\Delta \mathrm{F}}$ represented the instrument sensitiveness, the concentration $\mathrm{C}$ was invariable. In the range of $15-50{ }^{\circ} \mathrm{C}$, the heat of adsorption look upon as a constant, all data in the Table-1 being put into the formula 4. With to 1/T making diagram, the relation curve of the sensitivity of calixarene SAW sensor to the identical concentration was obtained under the different temperature condition (Fig. 2).

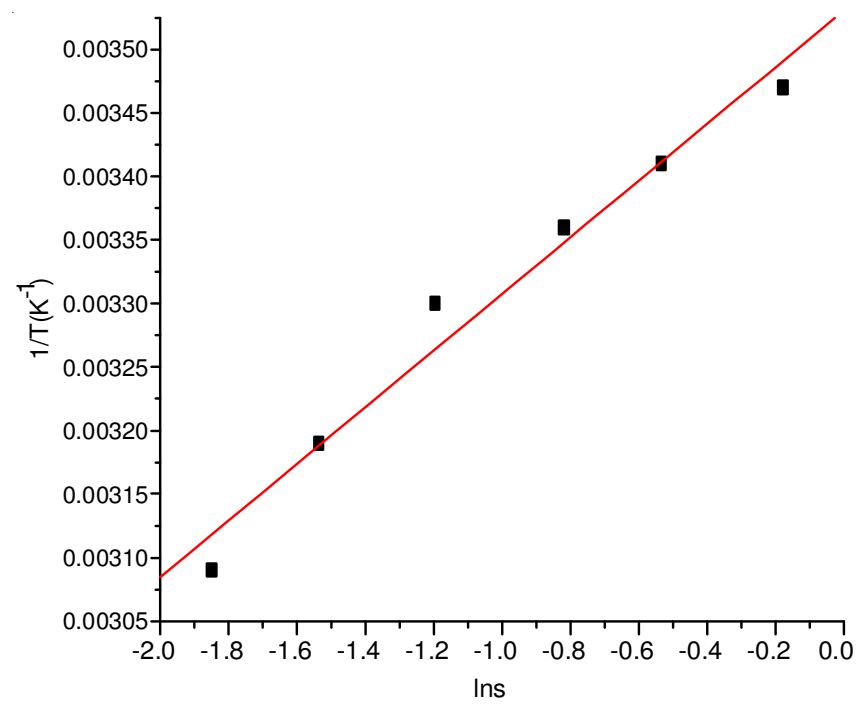

Fig. 2. Arrhenius plot of the SAW sensor sensitivity to the same concentration of sarin at difference temperature $(\mathrm{R}=0.9965$, $\mathrm{P}<0.0003)$

It could be seen from the Fig. 2 that the sensitiveness of calixarene-SAW sensor was consistent with negative Arrhenius equation temperature dependence relation. Along with the ascension (the range is a $15-50{ }^{\circ} \mathrm{C}$ ) of temperature, the respond frequency descended; therefore this process might be physicoadsorption process.

Moreover, under the above-mentioned experiment condition, the stability of the SAW sensor was checked with $20 \mathrm{mg} / \mathrm{m}^{-3}$ sarin. The four results of the experiment were basic 
homology. In spite of in the process of adsorbing or desorbing, the experimental results had fine reproducibility.

\section{Conclusion}

In the present work, the p-tert-butylcalix[4]arene selfassembly coatings on SAW sensor gold delay line have been prepared and the detailed research of detecting organic phosphorus compounds has carried out. Because this kind SAW-MIP sensor has strong selectivity, high sensitiveness, big respond frequency shift and restoring better to sarin it has fixed to detect the low concentration organic organophosphorus gas and has quickly early-warning potential and better of applied foreground. The discernment choosing capacity of molecule print technology and the were developed in an integral whole by the calixarene-SAW chemical sensors which have important drawing lessons meaning especially to study more gas sensitive film and sensors.

\section{ACKNOWLEDGEMENTS}

This work is partly supported by National Key Basic Research and Development Plan of China (No. 2011CB706902).

\section{REFERENCES}

1. F.F. Yang, Y.Y. Chen and S. Lin, Chin. J. Org. Chem., 22, 746 (2002).

2. B. Gao, Y.Q. Feng, L.S. Zhou and X. Ma, Chin. J. Org. Chem., 24, 713 (2004).

3. E.U. Thoden van Velzen, J.F.J. Engbersen and D.N. Reinhoudt, J. Am. Chem. Soc., 116, 3597 (1994).

4. K.D. Schierbaum, T. Weiss, E.U.T. van Veizen, J.F.J. Engbersen, D.N. Reinhoudt and W. Gopel, Science, 265, 1413 (1994).

5. B.T. Zhao, H.Y. Zhang and Y. Lui, Chin. J. Org. Chem., 25, 913 (2005).

6. E. Ghidini, F. Ugozzoli, R. Ungaro, S. Harkema, A. Abu El-Fadl and D.N. Reinhoudt, J. Am. Chem. Soc., 112, 6979 (1990). 